Table S1. Results of microbial testing of Centella asiatica botanical raw material samples evaluated for the manufacture of a Centella asiatica clinical trial product (CAP), Gotu kola preblend manufacturing intermediate and final products CAP $0 \mathrm{~g}$, CAP $2 \mathrm{~g}$ and CAP $4 \mathrm{~g}$.

CA-1 plant material (trade sample)

\begin{tabular}{|l|l|l|l|}
\hline Test & Supplier's data & OWH data & OWH Specifications \\
\hline Coliforms & N/A & $<10 \mathrm{cfu} / \mathrm{g}$ & Report only \\
\hline E.coli & Absent & $<10 \mathrm{cfu} / \mathrm{g} / \mathrm{not}$ detected & $<10 \mathrm{mpn} / \mathrm{g} /$ not detected \\
\hline Salmonella & Absent & Absent in $25 \mathrm{~g}$ & Absent in $25 \mathrm{~g}$ \\
\hline APC & $7885 \mathrm{cfu} / \mathrm{g}$ & $4,800 \mathrm{cfu} / \mathrm{g}$ & $<10,000,000 \mathrm{cfu} / \mathrm{g}$ \\
\hline Mold & Yeast+mold $854 \mathrm{cfu} / \mathrm{g}$ & $<100 \mathrm{cfu} / \mathrm{g}$ & $<100,000 \mathrm{cfu} / \mathrm{g}$ \\
\hline Yeast & Yeast+mold $854 \mathrm{cfu} / \mathrm{g}$ & $2,400 \mathrm{cfu} / \mathrm{g}$ & $<100,000 \mathrm{cfu} / \mathrm{g}$ \\
\hline
\end{tabular}

CA-2 plant material (trade sample)

\begin{tabular}{|l|l|l|l|}
\hline Test & Supplier's data & OWH data & OWH Specifications \\
\hline Coliforms & N/A & N/A & Report only \\
\hline E.coli & N/A & Conforms & $<10 \mathrm{mpn} / \mathrm{g} /$ not detected \\
\hline Salmonella & N/A & Conforms & Absent in $25 \mathrm{~g}$ \\
\hline APC & N/A & N/A & $<10,000,000 \mathrm{cfu} / \mathrm{g}$ \\
\hline Mold & N/A & N/A & $<100,000 \mathrm{cfu} / \mathrm{g}$ \\
\hline Yeast & N/A & $<100,000 \mathrm{cfu} / \mathrm{g}$ \\
\hline
\end{tabular}

CA-3 plant material (trade sample)

\begin{tabular}{|l|l|l|l|}
\hline Test & Supplier's data & OWH data & OWH Specifications \\
\hline Coliforms & $<100 \mathrm{cfu} / \mathrm{g}$ & $<10 \mathrm{cfu} / \mathrm{g}$ & Report only \\
\hline E.coli & Negative & $<10 \mathrm{cfu} / \mathrm{g} /$ not detected & $<10 \mathrm{mpn} / \mathrm{g} /$ not detected \\
\hline Salmonella & Negative & Negative & Absent in $25 \mathrm{~g}$ \\
\hline APC & $12,500 \mathrm{cfu} / \mathrm{g}$ & $200,700 \mathrm{cfu} / \mathrm{g}$ & $<10,000,000 \mathrm{cfu} / \mathrm{g}$ \\
\hline Mold & Yeast and mold $450 \mathrm{cfu} / \mathrm{g}$ & $<500 \mathrm{cfu} / \mathrm{g}$ & $<100,000 \mathrm{cfu} / \mathrm{g}$ \\
\hline Yeast & Yeast and mold $450 \mathrm{cfu} / \mathrm{g}$ & $600 \mathrm{cfu} / \mathrm{g}$ & $<100,000 \mathrm{cfu} / \mathrm{g}$ \\
\hline
\end{tabular}

CA-3 plant material (bulk sample)

\begin{tabular}{|l|l|l|l|}
\hline Test & Supplier's data & OWH data & OWH Specifications \\
\hline Coliforms & $<100 \mathrm{cfu} / \mathrm{g}$ & $<10 \mathrm{cfu} / \mathrm{g}$ & Report only \\
\hline E.coli & Negative & $<10 \mathrm{cfu} / \mathrm{g}$ & $<10 \mathrm{mpn} / \mathrm{g} /$ not detected \\
\hline Salmonella & Negative & Negative & Absent in $25 \mathrm{~g}$ \\
\hline APC & $12,500 \mathrm{cfu} / \mathrm{g}$ & $<5000 \mathrm{cfu} / \mathrm{g}$ & $<10,000,000 \mathrm{cfu} / \mathrm{g}$ \\
\hline Mold & Yeast and mold $450 \mathrm{cfu} / \mathrm{g}$ & $<500 \mathrm{cfu} / \mathrm{g}$ & $<100,000 \mathrm{cfu} / \mathrm{g}$ \\
\hline Yeast & Yeast and mold $450 \mathrm{cfu} / \mathrm{g}$ & $500 \mathrm{cfu} / \mathrm{g}$ & $<100,000 \mathrm{cfu} / \mathrm{g}$ \\
\hline
\end{tabular}

CA-6 plant material (trade sample)

\begin{tabular}{|l|l|l|l|}
\hline Test & Supplier's data & OWH data & OWH Specifications \\
\hline Coliforms & $100 \mathrm{cfu} / \mathrm{g}$ & N/A & Report only \\
\hline E.coli & Absent/10g & N/A & $<10 \mathrm{mpn} / \mathrm{g} / \mathrm{not}$ detected \\
\hline Salmonella & Negative/25g & N/A & Absent in $25 \mathrm{~g}$ \\
\hline APC & $50,000 \mathrm{cfu} / \mathrm{g}$ & N/A & $<10,000,000 \mathrm{cfu} / \mathrm{g}$ \\
\hline Mold & $100 \mathrm{cfu} / \mathrm{g}$ & N/A & $<100,000 \mathrm{cfu} / \mathrm{g}$ \\
\hline Yeast & $100 \mathrm{cfu} / \mathrm{g}$ & N/A & $<100,000 \mathrm{cfu} / \mathrm{g}$ \\
\hline
\end{tabular}


CA-6 plant material (bulk sample)

\begin{tabular}{|l|l|l|l|}
\hline Test & Supplier's data & OWH data & OWH Specifications \\
\hline Coliforms & $100 \mathrm{cfu} / \mathrm{g}$ & $>7000 \mathrm{cfu} / \mathrm{g}$ & Report only \\
\hline E.coli & Absent/10g & $<10 \mathrm{cfu} / \mathrm{g}$ & $<10 \mathrm{mpn} / \mathrm{g} / \mathrm{not}$ detected \\
\hline Salmonella & Negative/25g & Negative & Absent in $25 \mathrm{~g}$ \\
\hline APC & $50,000 \mathrm{cfu} / \mathrm{g}$ & $10,000 \mathrm{cfu} / \mathrm{g}$ & $<10,000,000 \mathrm{cfu} / \mathrm{g}$ \\
\hline Mold & $100 \mathrm{cfu} / \mathrm{g}$ & $<500 \mathrm{cfu} / \mathrm{g}$ & $<100,000 \mathrm{cfu} / \mathrm{g}$ \\
\hline Yeast & $100 \mathrm{cfu} / \mathrm{g}$ & $<500 \mathrm{cfu} / \mathrm{g}$ & $<100,000 \mathrm{cfu} / \mathrm{g}$ \\
\hline
\end{tabular}

CA-7 plant material (trade sample)

\begin{tabular}{|l|l|l|l|}
\hline Test & Supplier's data & OWH data & OWH Specifications \\
\hline Coliforms & $<10 \mathrm{cfu} / \mathrm{g}$ & N/A & Report only \\
\hline E.coli & $<10 \mathrm{cfu} / \mathrm{g}$ & N/A & $<10 \mathrm{mpn} / \mathrm{g} /$ not detected \\
\hline Salmonella & Negative & N/A & Absent in $25 \mathrm{~g}$ \\
\hline APC & $<100 \mathrm{cfu} / \mathrm{g}$ & N/A & $<10,000,000 \mathrm{cfu} / \mathrm{g}$ \\
\hline Mold & $<100 \mathrm{cfu} / \mathrm{g}$ & N/A & $<100,000 \mathrm{cfu} / \mathrm{g}$ \\
\hline Yeast & N/A & $<100,000 \mathrm{cfu} / \mathrm{g}$ \\
\hline
\end{tabular}

CA-8 plant material (trade sample)

\begin{tabular}{|l|l|l|l|}
\hline Test & Supplier's data & OWH data & OWH Specifications \\
\hline Coliforms & $<3 \mathrm{mpn} / \mathrm{g}$ & N/A & Report only \\
\hline E.coli & $<3 \mathrm{mpn} / \mathrm{g}$ & N/A & $<10 \mathrm{mpn} / \mathrm{g} / \mathrm{not}$ detected \\
\hline Salmonella & Negative/25g & N/A & Absent in $25 \mathrm{~g}$ \\
\hline APC & $380 \mathrm{cfu} / \mathrm{g}$ & N/A & $<10,000,000 \mathrm{cfu} / \mathrm{g}$ \\
\hline Mold & $<10 \mathrm{cfu} / \mathrm{g}$ & N/A & $<100,000 \mathrm{cfu} / \mathrm{g}$ \\
\hline Yeast & $<10 \mathrm{cfu} / \mathrm{g}$ & N/A & $<100,000 \mathrm{cfu} / \mathrm{g}$ \\
\hline
\end{tabular}

Gotu kola preblend (CAW spray dried extract blended from two batches)

\begin{tabular}{|l|l|l|}
\hline Test & OWH data & OWH Specifications \\
\hline Coliforms & $<10 \mathrm{cfu} / \mathrm{g}$ & Report only \\
\hline E.coli & $<10 \mathrm{mpn} / \mathrm{g}$ & $<10 \mathrm{mpn} / \mathrm{g} / \mathrm{not}$ detected \\
\hline Salmonella & Negative & Absent in $25 \mathrm{~g}$ \\
\hline APC & N/A & $<10,000,000 \mathrm{cfu} / \mathrm{g}$ \\
\hline Mold & N/A & $<100,000 \mathrm{cfu} / \mathrm{g}$ \\
\hline Yeast & N/A & $<100,000 \mathrm{cfu} / \mathrm{g}$ \\
\hline
\end{tabular}

CAP 0g

\begin{tabular}{|l|l|l|}
\hline Test & OWH data & OWH Specifications \\
\hline Coliforms & $<10 \mathrm{cfu} / \mathrm{g}$ & Report only \\
\hline E.coli & $<10 \mathrm{mpn} / \mathrm{g}$ & $<10 \mathrm{mpn} / \mathrm{g} /$ not detected \\
\hline Salmonella & Negative & Absent in $25 \mathrm{~g}$ \\
\hline APC & $<500 \mathrm{cfu} / \mathrm{g}$ & $<10,000,000 \mathrm{cfu} / \mathrm{g}$ \\
\hline Mold & $<500 \mathrm{cfu} / \mathrm{g}$ & $<100,000 \mathrm{cfu} / \mathrm{g}$ \\
\hline Yeast & $<500 \mathrm{cfu} / \mathrm{g}$ & $<100,000 \mathrm{cfu} / \mathrm{g}$ \\
\hline
\end{tabular}


CAP $2 \mathrm{~g}$

\begin{tabular}{|l|l|l|}
\hline Test & OWH data & OWH Specifications \\
\hline Coliforms & $<10 \mathrm{cfu} / \mathrm{g}$ & Report only \\
\hline E.coli & $<10 \mathrm{mpn} / \mathrm{g}$ & $<10 \mathrm{mpn} / \mathrm{g} /$ not detected \\
\hline Salmonella & Negative & Absent in $25 \mathrm{~g}$ \\
\hline APC & $<5000 \mathrm{cfu} / \mathrm{g}$ & $<10,000,000 \mathrm{cfu} / \mathrm{g}$ \\
\hline Mold & $<10,000 \mathrm{cfu} / \mathrm{g}$ & $<100,000 \mathrm{cfu} / \mathrm{g}$ \\
\hline Yeast & $<500 \mathrm{cfu} / \mathrm{g}$ & $<100,000 \mathrm{cfu} / \mathrm{g}$ \\
\hline
\end{tabular}

\section{CAP 4g}

\begin{tabular}{|l|l|l|}
\hline Test & OWH data & OWH Specifications \\
\hline Coliforms & $<10 \mathrm{cfu} / \mathrm{g}$ & Report only \\
\hline E.coli & $<10 \mathrm{mpn} / \mathrm{g}$ & $<10 \mathrm{mpn} / \mathrm{g} /$ not detected \\
\hline Salmonella & Negative & Absent in $25 \mathrm{~g}$ \\
\hline APC & $<500 \mathrm{cfu} / \mathrm{g}$ & $<10,000,000 \mathrm{cfu} / \mathrm{g}$ \\
\hline Mold & $<500 \mathrm{cfu} / \mathrm{g}$ & $<100,000 \mathrm{cfu} / \mathrm{g}$ \\
\hline Yeast & $<500 \mathrm{cfu} / \mathrm{g}$ & $<100,000 \mathrm{cfu} / \mathrm{g}$ \\
\hline
\end{tabular}

Suppliers data were taken from certificates of analysis; OWH = Oregon's Wild Harvest (Redmond, OR); "OWH data" were obtained by microbial testing in Oregon's Wild Harvest quality control laboratories; N/A = not available (data either not provided or not measured); $A P C=$ aerobic plate count; $c f u=$ colony forming units; $m p n=$ most probable number. 